\title{
Energy Efficient Load Balancing Strategy for Mobile Cloud Computing
}

\author{
Karanbir Singh \\ B.Tech. CSE \\ Guru Nanak Dev University \\ Amritsar
}

\begin{abstract}
Mobile Cloud Computing combines cloud computing and mobile computing. It has turned into one of the business trendy expressions and a real exchange string in the IT world. As MCC is still at the early phase of advancement, it is important to handle a careful understanding of the innovation to call attention to the bearing of future research. In this paper, a new algorithm is proposed load balancing for mobile clouds has been presented. The results has shown that the load balancing during job placement plays a critical role in energy consumption of cloud computing environment. The comparisons has clearly shown that the proposed technique outperforms over available techniques.
\end{abstract}

\section{Keywords}

Load Balancing, Mobile Cloud Computing

\section{INTRODUCTION}

With a capricious development of the portable applications and climbing of distributed computing idea, the Mobile Cloud Computing (MCC) has turned into a potential engineering for the versatile administration clients. MCC coordinates the innovation of distributed computing with versatile environment and has participated of premier talk string in the IT world since 2009.

Mobile Cloud computing as a coordination of distributed computing innovation with cell phones to make the cell phones asset full in states of computational force, memory, stockpiling, vitality, and connection readiness. Portable distributed computing is the after effect of interdisciplinary methodologies embodying mobile computing and cloud computing. Accordingly, this transdisciplinary field is otherwise called mobicloud processing.

\section{BACKGROUND}

This section presents the fundamental concepts of cloud computing and $\mathrm{MCC}$, and explains the theoretical aspects of application partitioning and distributed application processing for MCC.

\subsection{Mobile Computing}

Mobile administrations have picked up rate by the developing distributed computing advances, as these gadgets take an imperative part in the human life as both correspondence and stimulation, not limited by time and spot. The mobile computing (MC) gets to be compelling and quick in the improvement of IT engineering inside business and industry fields, also. Then again, the cell phones are confronting up with numerous battles in their assets (e.g., battery life, stockpiling, and data transmission) and interchanges (e.g., security, portability and security) [47]. These difficulties influence the change of administration qualities gravely.

Cloud computing (CC) began to be generally utilized and brought numerous open doors as a part of the method for assets like servers, systems, and stockpiles, stages and programming at low expenses. This convenience and minimal effort of CC can lead versatile applications to be more across the board and give assortment of administrations in the portable environment. The versatile applications can be thought another route from the cloud suppliers' point of view; it can be coordinated with the current cloud framework without requiring any extra expensive foundation, yet new sorts of administrations and offices for the portable clients.

The Mobile Cloud Computing (MCC) term was presented after the idea of Cloud Computing. Fundamentally, MCC alludes to a foundation where both the information stockpiling and the information transforming happen outside of the cell phone [21]. With respect to definition, versatile applications move the reckoning force and capacity from the cellular telephones to the cloud. It can be thought as an issue of the distributed computing and versatile environment. The cloud can be utilized for force and capacity, as cell phones don't have compelling assets contrasted with customary reckoning gadgets.

Today, there are as of now heaps of great cases of MCC applications including Gmail, Google Maps, and such applications. In any case, a large portion of uses still do the vast majority of the estimation and information stockpiling on the cell phones themselves, not in the cloud. In a couple of years, that could change [36].

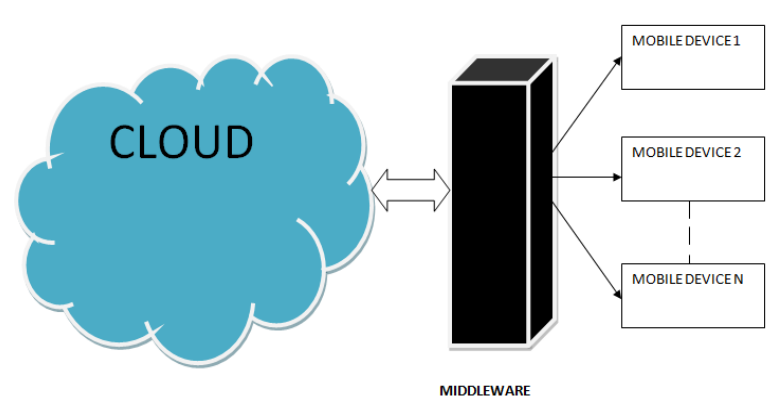

Fig 1: Mobile Cloud Computing

\subsection{Mobile Cloud Computing Architecture} a) Access Layer

Access layer moreover well-known as an issue layer (application layer) is at risk to give coordinated effort between the client and the cloud. The organization interface, enlistment and organization get all the fragments. It institutionalizes all principles, finishing the enrollment of client or administration.

\section{b) Management Layer}

The administration (stage) layer of the construction modeling is in control for giving administration benefits between server groups, and the administrations asked by the cell phone. It gives standard conventions, interfaces, notice, security, 
honesty, affirmations and standard strategy of procedure in the cloud. Since there are numerous distinctive stages or working frameworks in the cell phones, demanding capacity to emit the working framework contrast is an obligation of the administration layer. It takes inconvenience of charging framework, environment, account setup, errand portion, assignment administration, undertaking execution and lifecycle administration.

\section{c) Virtual Layer}

Virtual (base) layer implies virtual things, for example, computing pool, stockpiling pool and network pool. Virtual capacities can be actualized by programming capacity, including virtual environment, virtual framework and virtual stage.

\section{d) Physical Layer}

The physical (equipment) layer gives the actualities of the physical gadgets, for example, desktop machines, cell telephones, tablets, advanced mobile phones, flimsy purchasers or imbecilic telephones.

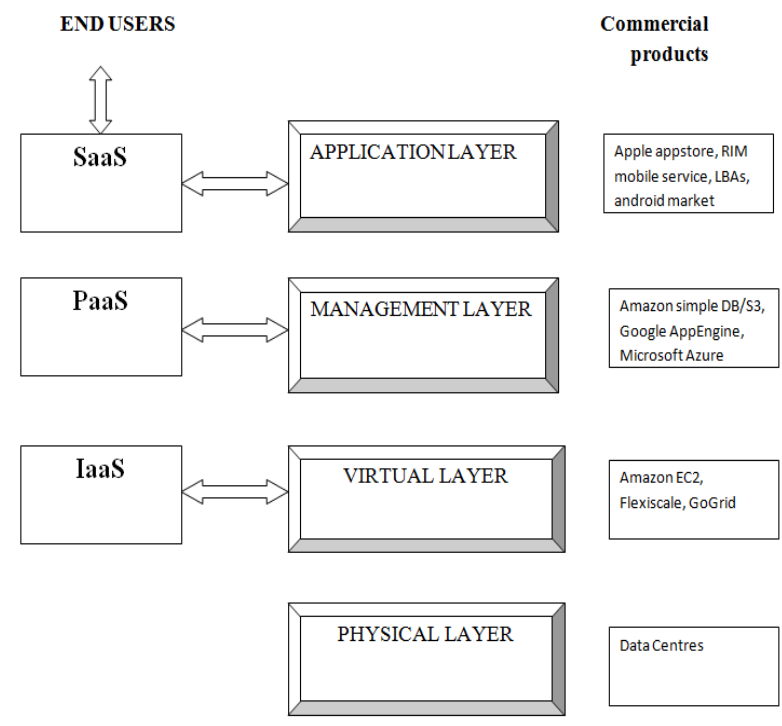

Fig 2: shows architecture of MCC

By and large, a distributed computing is an extensive scale appropriated system framework executed focused around various servers in server farms. The cloud administrations are for the most part ordered focused around a layer idea. In the upper layers of this ideal model, Infrastructure as a service (IaaS), Platform as a service (PaaS), and Software as a service (SaaS) are stacked.

-Data focuses layer: This layer gives the fittings office and framework for mists. In server farm layer, various servers are connected with fast systems to give administrations to clients. Normally, server farms are inherent less populated spots, with high power supply soundness and an okay of catastrophe.

- Infrastructure as a service (IaaS): IaaS is focused around top of the server layer. IaaS enables the obtainment of limit, fittings, servers and frameworks organization sections. The client routinely pays on a for each usage premise. Along these lines, clients can extra cost as the portion is simply concentrated around the measure of advantage they really usage. Establishment can be expanded or contracted logically as needed. The instances of IaaS are Amazon EC2 (Elastic Cloud Computing) and S3 (Simple Storage Service)
- Platform as a service (PaaS): PaaS offers an advanced facilitated environment for building, testing and sending custom applications. The instances of PaaS are Google App Engine, Microsoft Azure, and Amazon Map Reduce/Simple Storage Service.

-Software as a service (SaaS): SaaS helps in product dissemination with particular necessities. In this layer, the clients can get to an application and data remotely through the Internet and pay just for that they utilize. Salesforce is one of the pioneers in giving this administration model. Microsoft's Live Mesh likewise permits offering records and organizers crosswise over various gadgets at the same time.

Although the cloud computing architecture can be divided into four layers. It doesn't imply that the top layer must be based on the layer specifically underneath it. Case in point, the SaaS application can be sent specifically on IaaS, rather than PaaS. Likewise, a few administrations can be considered as an issue of more than one layer. Case in point, information stockpiling administration can be seen as either in IaaS or PaaS. Given this structural model, the clients can utilize the administrations adaptably and effectively.

\subsection{Mobile Cloud Computing Advantages/Disadvantages}

There are numerous motivations to utilize distributed computing with portable applications. MCC gives a few answers for the obstructions which portable endorsers are normally face up with. These focal points are:

\section{a) Battery Life}

Battery life is one of the primary concerns in the versatile environment. There are now a few answers for developing battery life by improving CPU execution, utilizing plate and screen as a part of a proficient way to diminish power utilization. Anyhow these arrangements for the most part oblige changes in the cell phones structure or other fittings which means expanding the expense. Calculation or information offloading procedures are proposed to relocate the immense and complex calculations from restricted asset gadgets like cell phones to effective machines like servers in mists. [35].

\section{b) Data stockpiling limit/Process power}

An alternate obstruction is capacity limit of cell phones. Cell phones are by and large have constrained capacity. To defeat this issue, MCC can be utilized to get to, question or store the substantial information on the cloud through remote systems. There are a few illustrations which are generally utilized, for example, Amazon Simple Storage Service (Amazon S3) to give document stockpiling on the cloud.

\section{c) Reliability}

With the assistance of CC ideal model, unwavering quality can be enhanced since information and applications are hidden away moved down on a few quantities of machines on the cloud. This gives more classifiedness by diminishing the shot of information lost on the cell phones. Also, copyrighting computerized substance and anticipating illicit disseminations like music, feature can be more accessible in this model. Additionally security administrations like infection identification applications can be effortlessly given and utilized as a part of an effective path without effecting cell phone execution. Moreover, CC adaptability, flexibility preferences can be utilized as a part of MCC, too since cloud adaptability is pertinent as an issue base, in the same way. 


\section{d) Privacy}

Assurance is a vital issue, when contemplating private data. As in the CC time, the same trust issue turns out with the adaptable framework suppliers and cloud suppliers. They can screen at all the correspondence and data set away in the cloud or framework supplier, in spite of the way that there is encryption frameworks to grave data passed on or set away. So from this perspective, it is an enormous headache to be clarified.

\section{e) Communication}

The correspondence is formed from numerous parts from portable endorser of the cloud supplier. Along these lines there can be a few issues like poor system pace or 7 restricted transfer speed. It can be an enormous concern on the grounds that the quantity of versatile and cloud clients is significantly expanding.

\subsection{Challenges In Mobile Cloud Computing}

As said in the past segment, Mobile Cloud Computing has numerous profits and great application samples for portable clients and administration suppliers. Then again, as specified in a few parts, there are likewise a few difficulties identified with distributed computing and portable systems correspondence. This area provides for some clarification about these deterrents and arrangements.

\subsubsection{Mobile Side Challenges}

In the Mobile system side, principle impediments and arrangements are recorded underneath:

\section{a) Low Bandwidth}

Data transfer capacity is the one of imperative issues in portable cloud environment on the grounds that versatile system asset is much littler contrasted and the customary systems. Thusly, P2P Media Streaming for offering constrained data transfer capacity among the clients who are found adjacent in the same range for the same substance, for example, the same feature [29]. By this system, every client transmits or trades parts of the same substance with alternate clients, which is brought about change of substance quality, particularly for features.

\section{b) Availability}

System disappointments, out of sign slips, or high activity related poor execution issues are principle dangers to counteract clients to join with the cloud. Yet there are a few answers for help portable clients on account of any disengagement from the mists. One of them is Wi-Fi Based Multihop MANET. It is an appropriated substance offering convention for the circumstances without any foundation . In this system, adjacent hubs are caught if there should be an occurrence of the disappointment of immediate association with the cloud. For this situation, as opposed to having a connection specifically to the cloud, portable client can interface with the cloud through neighboring hubs. Despite the way that there are a couple of considers about security issues for such frameworks, these issues can in like manner be lit up.

\section{c) Heterogeneity}

There are sorts of systems which are utilized all the while as a part of portable environment, for example, WCDMA, GPRS, Wimax, Cdma2000, and WLAN. As an issue, taking care of like heterogeneous system network gets to be hard while fulfilling versatile cloud heterogeneous system integration gets to be hard while fulfilling portable distributed computing prerequisites, for example, network which is constantly on, on-interest adaptable integration, and the vitality productivity of cell phones. This issue can be comprehended by utilizing institutionalized interfaces and informing conventions to achieve, oversee and disperse substance.

\section{d) Pricing}

Using distinctive organizations as a piece of versatile requires with both adaptable framework supplier and cloud organization supplier. Of course, these suppliers have different procedures for portion and expenses for organizations, contrivances and work places. Thus, this has likelihood of provoking various issues like how to center expense, how the expense could be granted among the suppliers or parties, and how the endorsers can pay. As a case, when a compact customer needs to run a not free adaptable application on the cloud, this joins in three stakeholders as one of them is application supplier for application grant, second one is versatile framework supplier for used data correspondence from customer to cloud, and third one is cloud supplier for giving and running application on the cloud.

\section{Computing Side Challenges}

In the cloud side, fundamental obstructions and arrangements are recorded underneath:

\section{a) Computing Offloading}

As specified past parts, offloading is one of the key gimmicks of MCC to enhance the battery life time and to expand the applications' execution by utilizing the cloud [32]. Despite the fact that this arrangement is extremely valuable in the method for methodology force and capacity, it can be inadequate in a few circumstances. Case in point, the cell phones can devour more vitality for an application by utilizing the cloud instead of neighborhood transforming. For a discriminating edge, utilizing the cell phone rather than cloud may be more powerful.

Accordingly, an issue emerges that the ideal method for exchange off between the correspondence and computation expenses must be assessed or ascertained for portable applications. The correspondence cost mostly relies on upon the measure of transmitted information and the transfer speed of system, while the processing expense can be characterized by the method for calculation time. The ideal choices of a system apportioning can be made at a runtime powerfully, by utilizing and working expense calculations [39].

As an example, a philosophy for picking which parts of Java ventures should be offloaded can be given. In this approach, a Java undertaking is differentiated into methodologies and uses a couple of parameters like size of strategies or line of codes to figure execution costs for these schedules. By then, this system takes a gander at the close-by execution costs of each method with the remote (cloud) execution costs to settle on a perfect execution decision [32].

\section{b) Security}

Trust is the principal issue of the endorsers in the convenient stage. Concerning MCC, this issue grabs more centrality as the stakeholders increase in the cloud environment for guaranteeing customer insurance and data/application puzzle. One of the security issues is wireless customers, other one is the data insurance and security.

As versatile clients, there are a few security dangers like malignant codes like infection, worm, and Trojan steeds and protection concerns when considering coordinated worldwide situating framework (GPS) gadgets that can lead endorsers of 
be followed. To defeat this issue, there is security projects can be run on cell phones to forestall unlawful dangers, yet these projects utilize huge parcel of cell phone assets while running. Thusly there are a few methodologies moves danger recognition abilities from cell phones to cloud. For instance, Cloud AV stage gives a different administration focused around both cloud and cell phone for malware recognition [10]. A fundamental and lightweight bit of the application runs on the SMD and it compares with the genuine portion of the application in the cloud. Disclosure limits are moved to the cloud, as the versatile expert's application sends record parts beyond anyone's ability to see to the cloud server application.

\section{c) Authentication}

Yet both application architects and compact customers advantage from securing and changing a great deal of data/applications on a cloud, they should be watchful of dealing with the data or applications in regards to rights and check. Customers have penchant of using little passwords while getting to outside resources, so there is prerequisite for more secure confirmation frameworks. As a delineation of confirmation framework, Trustcube can be given. It is a course of action based cloud check instrument using open benchmarks and it joins distinctive sorts of affirmation [5].

IA Server

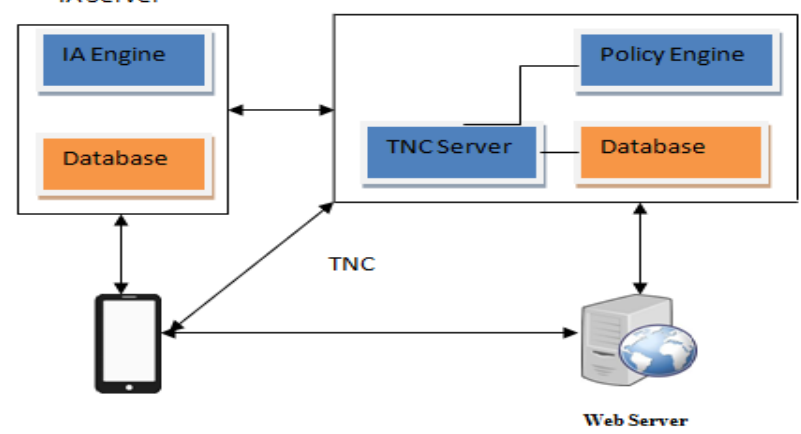

Fig 3: The Trust Cube architecture, one implementation of the authentication framework [5]

As in the figure 3, the framework building design shows clients security for confirmation for getting to assets. At the point when a web server gets an appeal from the cell phone, web server sends solicitation to Integrated Authenticated Service (IAS) with the points of interest of solicitation. At the point when IAS gets the solicitation, extricates data and control the approaches of the message. It sends a request to the IA Server over a safe trusted system association convention (TNC). IA Server takes the request and reacts to IAS with a produced report about the verification guidelines. IAS decides confirmation come about, and sends this result to the web server. As an issue, the client can be confirmed or not, toward the end.

\section{d) Data Access}

While cloud administrations are expanding, the quantity of information assets on the cloud quickly increment. Hence, managing these information assets in the method for putting away, overseeing or getting to gets to be exceptionally difficult. Distributed storage suppliers like Amazon S3, each information yield operations executes employments for the most part, in document level which expands the expense of information correspondence and handling for portable customers. There are a few arrangements that giving a productive and less expensive method for square level based
I/O calculations rather than record level. In this arrangement, not all the document transmitted, rather information pieces are transmitted, if there should arise an occurrence of need, which is exceptionally helpful by the method for time and system correspondence cost.

Likewise, to expand information access proficiency, cell phones can utilize nearby capacity as reserve for particular parts of cloud administration to build access speed and diminish system need. Here, there is likewise an issue, as we can't store vast information on the cell phone stockpiling reserve, so some information administration or information choices components must be connected to figure out which parts or measure of information can be reserved or utilized from cloud.

\section{PROPOSED MULTI QUEUE JOB SCHEDULING}

The proposed approach enhances the scheduler grouping the various burst time based jobs into queue. The client submitted jobs sorted based on the ascending order of the burst time and it gives equal importance to all jobs. It helps to increase the client satisfaction because the client requirements are varying based on the current needs. It reduces starvation using the dynamic allocation of jobs to select the best suitable jobs from among the available and does not decrease the performance of the system

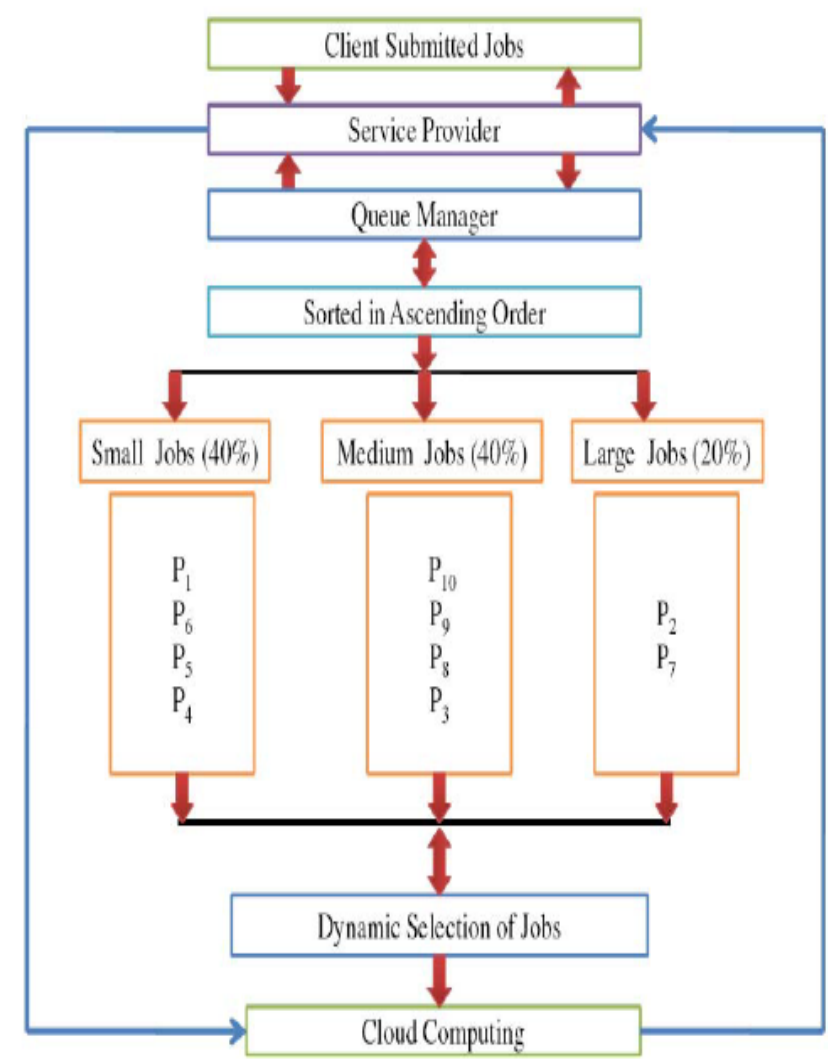

Fig 4: MQS Algo.

The queue manager provides the resources for the underlying network. The queue manager is a part of the cloud computing which manages the utilization of all the resources in the cluster. It keeps the track of the systems, which are all currently running the jobs by balancing the load among the metascheduler and its disposal. It indicates the scheduler to schedule the output of the job which is collected back by the queue manager. The scheduling process and resource 
allocation process is based on real time scheduling with unpredictable events. Three different queues are formed as small, medium and long based on ascending order measured in terms of burst time of the jobs.

1. In the small queue first $40 \%$ of jobs are stored.

2. In the medium queue next $40 \%$ of jobs are stored.

3. In the long queue remaining $20 \%$ jobs are stored.

The proposed method gives importance to all the jobs because many number of client in cloud computing and each client wants more needs and expectation. It grants equal importance for all in dynamic selection. The following Fig 1.shows the architecture of MQS using dynamic selection of jobs based on the sorted burst time. The client submitted jobs are enter in to the service provider it consists of queue manager that sort the jobs in ascending based on burst time. The jobs are executed based on dynamic selection and enter in to cloud environment. The best allocation reduces the time and availability of space in an effective manner without compensating the quality of the system and customer needs. The scheduler takes job id P1 and P6 from small queue and job id P10 and P9 from medium queue and job id $\mathrm{P} 2$ from long queue correspondingly and repeats for all jobs.

1st Iteration $\mathrm{P} 1 \rightarrow \mathrm{P} 6 \rightarrow \mathrm{P} 10 \rightarrow \mathrm{P} 9 \rightarrow \mathrm{P} 2$

2nd Iteration P5 $\rightarrow$ P4 $\rightarrow$ P8 $\rightarrow$ P3 $\rightarrow$ P7

Enhanced MQS scheduling using fuzzy if then rules that will enhance the load balancing based on fuzzy values.

Following are the steps of proposed methodology are as:
a. Initialize cloud.
b. Initialize load.
c. Balance load among cloud nodes.
d. Check load frequently.
e. Evaluate fuzzy score for live migration.

f. Is fuzzy score greater than threshold value, then live migration is carried out. But if fuzzy score is less than threshold value, then again balance load among cloud nodes.

\section{RESULTS AND DISCUSSIONS}

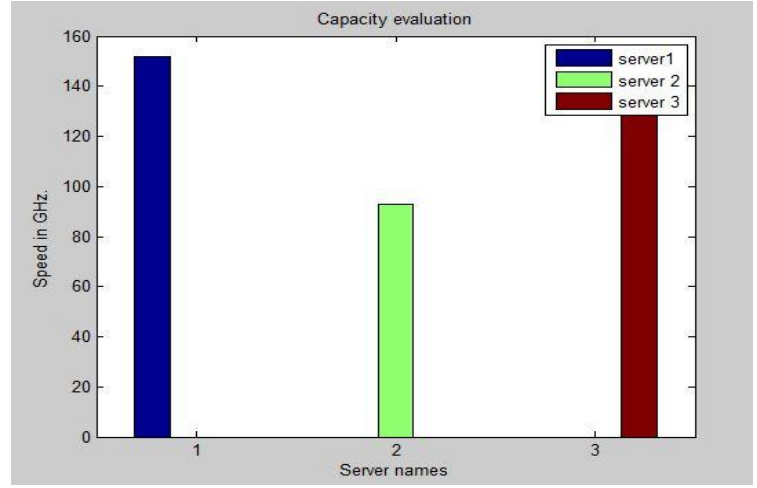

Fig 3: In this graph, we evaluate the capacity of three different servers. The cloud will request which have highest capacity. So, the cloud will give priority to the server "1" , then it will give priority to server " 3 ".
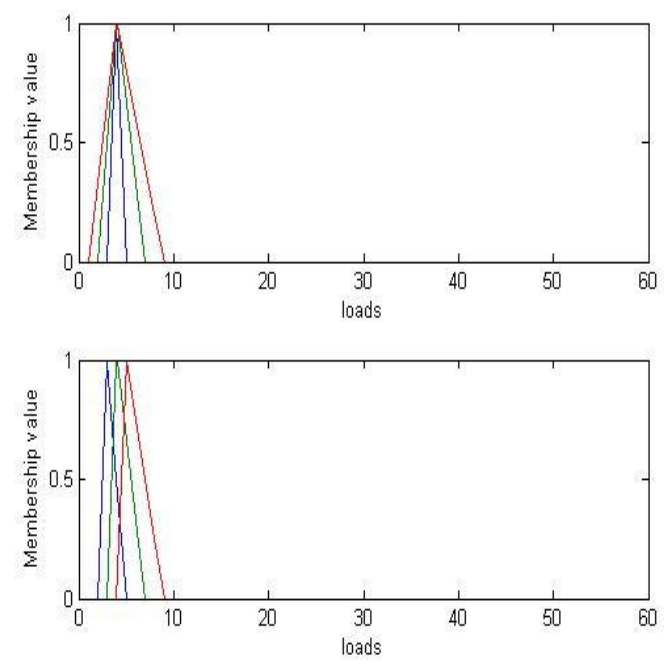

Fig 4: Fuzzy rules

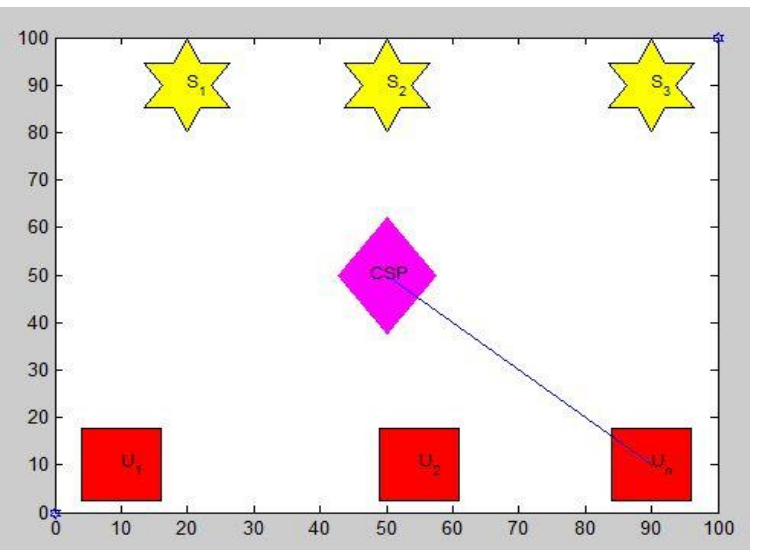

Fig 5: This graph shows that there are U1, U2,.......... Un are "N" no. of users. Similarly, S1, S2, S3 are three different servers. There is carrier service provider [CSP] which manages the utilization of the resources. One of the users, send request to carrier service provider [CSP] for required resource. Then CSP will check that which server has highest capacity and compatible with user's requirement, then CSP will allocate that server to requested user. At one instant, one user can request to CSP.

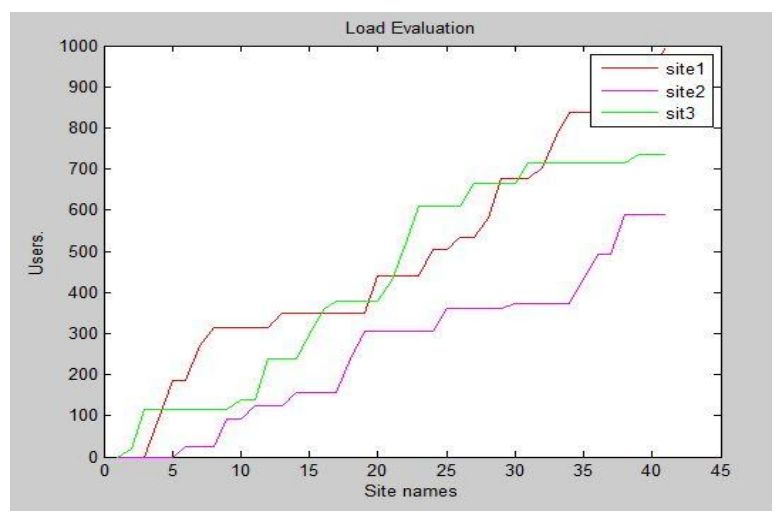

Fig 6: This graph evaluates the load on three different sites i.e. site1, site 2, site 3. Some load is added to the sites and some load is matured on any instant. As the number of users increases, load also increases. 


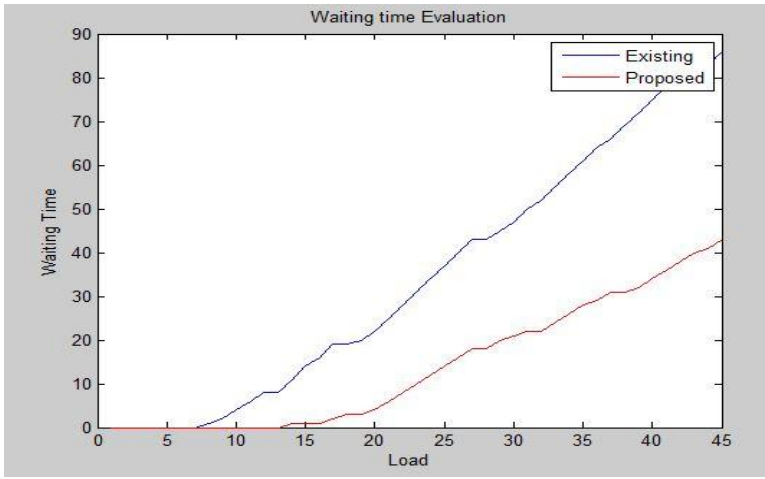

Fig 7: This graph compares the waiting time of proposed algorithm with exiting algorithm. Waiting time is the enough time that the process remains in the ready queue. The proposed algorithm has lesser waiting time than exiting algorithm. So, lesser waiting time means proposed algorithm operates jobs more quickly than exiting algorithm. This comparison result shows that proposed algorithm is efficient and better than exiting algorithm.

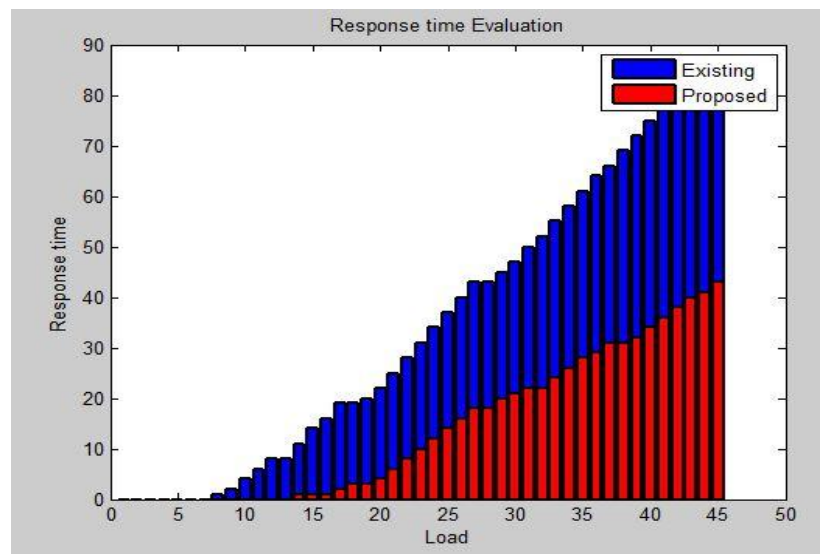

Fig 8: This graph compares the response time of proposed algorithm with exiting algorithm. Response time is the timeframe required from whenever a request was submitted before first response is produced. The proposed algorithm has lesser response time than exiting algorithm. So, lesser response time means proposed algorithm operates jobs more quickly than exiting algorithm. This comparison result shows that proposed algorithm is efficient and better than exiting algorithm.

\section{CONCLUSION}

As cloud computing is picking up prevalence, an imperative inquiry is the means by which to ideally convey programming applications on the offered base in the cloud. Especially in the setting of mobile computing where programming parts could be offloaded from the cell phone to the cloud, it is vital to improve the association, by minimizing the framework utilization. This work improved the prevailing function through presenting the actual energetic awaken cell phone calls with the idea to banned the actual dynamic hosts or system the actual passive server. This wakeup get in touch with are going to be initiated as soon as the body weight can be $80 \%$ as well as shut down are going to be initiated whenever we re-allocate insert derived from one of hosting server so that you can other. The overall particular attention can be to lower the actual result period of end users which is to be elevated through wakeup period in pre-existing analysis work. As a result we will make the most of the ability intake proficiently and even reduce the result period of pre-existing work.

\section{REFERENCES}

[1] Karthick, A. V., E. Ramaraj, and R. Ganapathy Subramanian. "An Efficient Multi Queue Job Scheduling for Cloud Computing." In IEEE World Congress Computing and Communication Technologies, pp. 164166. IEEE, 2014.

[2] Tiwari, Mangal Nath, Kamalendra Kumar Gautam, and D. R. Katare. "Analysis of Public Cloud Load Balancing using Partitioning Method and Game Theory."International Journal of Advanced Research in Computer Science and Software Engineering 4, no. 2 (2014).

[3] Kumar, Vikas, and Shiva Prakash. "A Load Balancing Based Cloud Computing Techniques and Challenges." International Journal of scientific research and management, Volume||2||Issue||5 ||Pages|| 815-824 ||2014|

[4] Sahu, Yatendra, and R. K. Pateriya. "Cloud Computing overview with load balancing techniques." International Journal of Computer Applications Vol. 65, no. 24 (2013).

[5] N. Sasikala and Dr. D. Ramesh, "Effective Load Balancing for Cloud Computing using Hybrid AB Algorithm", International Journal of Innovative Research in Computer and Communication Engineering, Vol. 2, Issue 4, April 2014

[6] Shah, MR Manan D., MR Amit A. Kariyani, and MR Dipak L. Agrawal. "Allocation Of Virtual Machines In Cloud Computing Using Load Balancing Algorithm." International Journal of Computer Science and Information Technology \& Security (IJCSITS), Vol. 3, No.1, February 2013.

[7] Priya, S. Mohana, and B. Subramani. "A New Approach For Load Balancing In Cloud Computing." International Journal of Engineering and Computer Science, Volume 2 Issue 5 May, 2013.

[8] S. Adiseshu Gupta, K. V. Srinivasa Rao, "Implementation of Load Balancing Based on Partitioning in Cloud Computing", International Journal of Advanced Research in Electrical, Electronics and Instrumentation Engineering, Vol. 3, Issue 10, October 2014.

[9] Ruhi Gupta. "Review on Existing Load Balancing Techniques of Cloud Computing." International Journal of Advanced Research in Computer Science and Software Engineering Volume 4, Issue 2, February 2014.

[10] Gulati, Ajay, and Ranjeev K. Chopra. "Dynamic round robin for load balancing in a cloud computing." International Journal of Computer Science and Mobile Computing, Vol. 2, Issue. 6, June 2013.

[11] Ray, Soumya, and Ajanta De Sarkar, "Execution analysis of load balancing algorithms in cloud computing environment" International Journal on Cloud Computing: Services and Architecture Vol.2, No.5, October 2012.

[12] Mishra, Ratan, and Anant Jaiswal. "Ant colony optimization: A solution of load balancing in cloud." International Journal of Web \& Semantic Technology Vol.3, No.2, April 2012. 
[13] Raghava, N. S., and Deepti Singh. "Comparative Study on Load Balancing Techniques in Cloud Computing." Open Journal of Mobile Computing and Cloud Computing, Volume 1, Number 1, August 2014.

[14] Kansal, Nidhi Jain, and Inderveer Chana. "Cloud load balancing techniques: a step towards green computing." IJCSI International Journal of Computer Science, Vol. 9, Issue 1, No 1, January 2012.

[15] Amandeep, Vandana Yadav, Faz Mohammad, "Different Strategies for Load Balancing in Cloud Computing Environment: a critical Study", International Journal of Scientific Research Engineering \& Technology, Volume 3 Issue 1, April 2014.
[16] Tejinder Sharma, Vijay Kumar Banga, "Efficient and Enhanced Algorithm in Cloud Computing", International Journal of Soft Computing and Engineering, Volume-3, Issue-1, March 2013.

[17] Miss. Neeta S. Nipane Prof. Nutan M. Dhande, “ABC LOAD BALANCING TECHNIQUE - IN CLOUD COMPUTING", International Journal of Innovative Research in Advanced Engineering, Volume 1, Issue 2 (April 2014).

[18] Shagufta khan 2Niresh Sharma, "Ant Colony Optimization for Effective Load Balancing In Cloud Computing", International Journal of Emerging Trends \& Technology in Computer Science, Volume 2, Issue 6, November - December 2013. 